\title{
Noise Reduction Using Compensation Capacitance for Bend Discontinuities of Differential Transmission Lines
}

\author{
Guang-Hwa Shiue, Wei-Da Guo, Chien-Min Lin, Member, IEEE, and Ruey-Beei Wu, Senior Member, IEEE
}

\begin{abstract}
Differential signaling has become a popular choice for multigigabit digital applications in favor of its low-noise generation and high common-mode noise immunity. Recalling from the full-wave solution of $S$-parameters, this paper presented a design methodology of analysis scheme to extract the equivalent circuits of discontinuities observed on the strongly coupled differential lines. Signal integrity effects of the bent differential transmission lines in a high-speed digital circuit were then simulated in the time domain. A dual back-to-back routing topology of bent differential lines to reduce the common-mode noise was further investigated. To alleviate the common-mode noise at the receiver, a novel compensation scheme in use of the shunt capacitance was also proposed. Furthermore, the comparison between the simulation and measured results validated the equivalent circuit model, coupled bends with compensation capacitance patch, and analysis approach.
\end{abstract}

Index Terms-Common-mode noise, compensation capacitance, differential signaling, discontinuity, dual back-to-back coupled bends, signal integrity, strongly coupled differential line, mixed-mode $S$-parameters.

\section{INTRODUCTION}

W ITH the increasing density of digital circuit layouts and faster pulse edges, the bend discontinuities of microstrip and stripline structures have now been shown to introduce noticeable signal degradations for short rise-time pulses. In the past, various single-line bends have been investigated on the $S$-parameters [1], [2], lumped element equivalent circuit model extraction [3], [4], and radiation effect [5], [6]. Considering the serial data links for high-speed multigigabit per second digital circuitry (such as IEEE 1394, USB 2.0, Serial ATA, and PCI-Express), differential signaling is usually used to improve the signal integrity and reduce the electromagnetic interference (EMI). The routing scheme is in favor of its low-noise generation and high common-mode noise immunity.

For an ideal serial data link, the differential interconnect maintains the differential symmetry and, therefore, the differen-

Manuscript received April 8, 2005; revised October 15, 2005. This work was supported in part by the National Science Council of Taiwan, R.O.C., under Grant NSC 91-2213-E-002-109, in part by the Ministry of Education under Grant 93B-40053, in part by Inventec under Grant $93-S-B 10$, and in part by Taiwan Semiconductor Manufacturing Company, Ltd. under Grant 93-FS-B072.

G.-H. Shiue, W.-D. Guo, and R.-B. Wu are with the Department of Electrical Engineering and Graduate Institute of Communication Engineering, National Taiwan University, Taipei 10617, Taiwan, R.O.C.

C.-M. Lin is with the Packaging Core Competence Department, Advanced Assembly Division, Taiwan Semiconductor Manufacturing Company, Ltd., Hsinchu 310, Taiwan, R.O.C.

Color versions of Figs. 1-11 and 13 are available online at http://ieeexplore.ieee.org.

Digital Object Identifier 10.1109/TADVP.2006.875413 tial to common-mode conversion is not an issue. In a practical layout scenario, the interconnect topology is nonetheless asymmetrical due to the bends, presence of adjacent traces, and physical constraints in routing near the package and connectors. The impact of signal integrity and EMI [7] on the amount of common-mode noise generated by the phase skew of different routing lengths should be considered as well.

In recent years, various methods have been adopted to model a multiconductor microstrip using the $\mathrm{T}$ or $\pi$ model, such as the method of moments [8], method of lines [9], analysis with the efficient excess-charge and excess-current techniques [10], model extraction from the time-domain reflection/transmission (TDR/T) measurement method [11], and three-dimensional (3-D) transmission-line matrix method [12]. However, these papers did not investigate the common-mode noise induced by differential bends and noise reduction scheme. In this paper, the feasible design methodology is hereby proposed to explore the signal integrity analysis for the discontinuity structures according to the growing demands on high-speed interconnects.

Starting from the calculated $S$-parameters of strongly coupled differential lines by the full-wave solver IE3D, ${ }^{1}$ the equivalent circuit model of bend discontinuities is extracted as described in Section II. Various bent differential transmission lines in a high-speed digital circuits are outlined in Section III. Their signal integrity effects are then analyzed in the time domain to characterize the parasitic data and common-mode and differential-mode noise profiles. As a noise reduction scheme in Section IV, both the dual coupled bends design and the detour design for reducing the common-mode noise in its microstrip structure are investigated. A novel compensation scheme using the shunt capacitance is proposed to alleviate the common-mode noise at the receiver. In Section V, favorable comparisons between the simulation and measurement results in the time-domain and frequency-domain validate the equivalent circuit model and analysis approach. The overview summary for this paper is in Section VI.

\section{THEORY AND CIRCUIT MODELING}

\section{A. Model Setup}

To investigate the signal integrity issues of bend discontinuities, it is necessary to develop the lumped and frequency-dependent equivalent circuits for these configurations. Considering the bend discontinuity of differential lines for its layout size to be much smaller than the wavelength of operating frequency

${ }^{1}$ IE3D. Zeland Software, Inc., www.zeland.com 


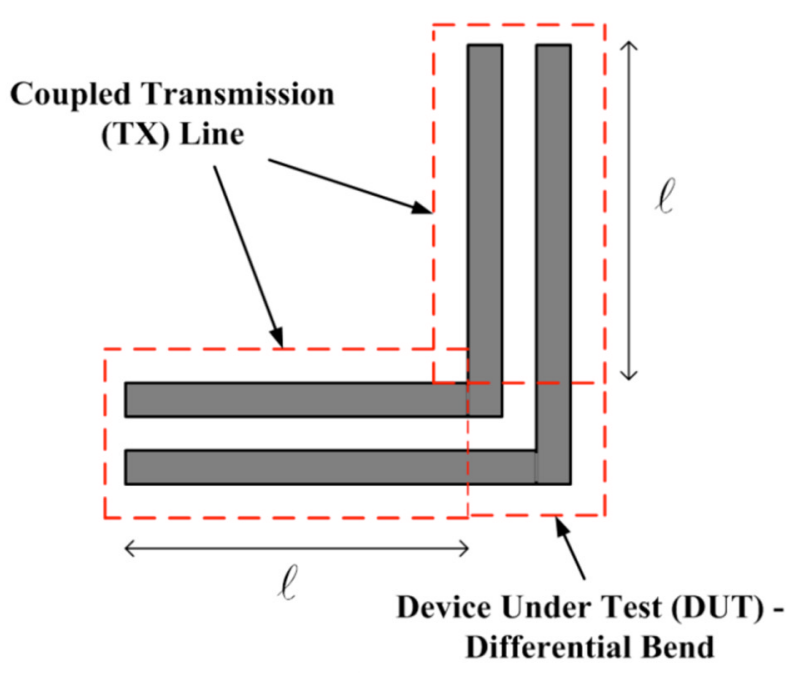

(a)

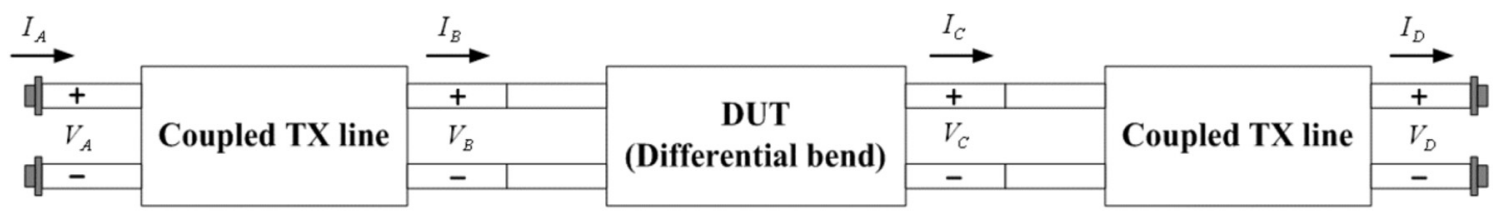

(b)

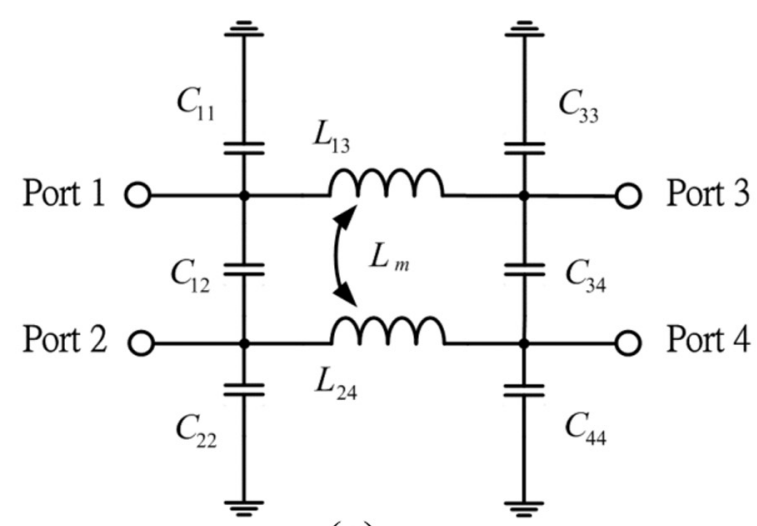

(c)

Fig. 1. Graphical representation of bent coupled transmission lines. (a) Physical layout. (b) Block diagram. (c) Lumped equivalent circuit model.

range as depicted in Fig. 1(a), the block diagram of coupled transmission lines can be modeled as shown in Fig. 1(b), where the bend can be approximated by a simple lumped circuit in reference to Fig. 1(c). This lumped circuit model is an extension of the $\pi$ model used for a single transmission line. Though this model can include the radiation, conductor and dielectric losses, and frequency-dependent resistance, these losses are small and negligible for practical applications up to gigabit per second rates.

\section{B. De-Embedding Tx Line Effects}

Most of the present commercial electromagnetic field solvers assume the isolated output ports in deembedding the transmission line effects. However, when the input and output transmission lines are strongly coupled, a more general approach needs to be developed. For simplicity, a system of $N$ bent coupled transmission lines is considered here. By using the field solvers, its full-wave characteristics can be modeled in terms of a $2 N$-port $A B C D$ matrix. Based on the cascade property, the $A B C D$ matrix can be divided into three parts: two sections of coupled transmission lines and a bend structure between.

For the block diagram as depicted in Fig. 1(b), the $A B C D$ matrix of bent differential transmission lines at the output port $D$ in response to the input port $A$ can be formulated as

$\left[\begin{array}{c}\vec{V}_{D} \\ \vec{I}_{D}\end{array}\right]=\left[\begin{array}{cc}\vec{A}_{\ell} & -\vec{B}_{\ell} \\ -\vec{C}_{\ell} & \vec{D}_{\ell}\end{array}\right]^{-1} \cdot\left[\begin{array}{ll}\vec{A}_{b} & \vec{B}_{b} \\ \vec{C}_{b} & \vec{D}_{b}\end{array}\right] \cdot\left[\begin{array}{cc}\vec{A}_{\ell} & \vec{B}_{\ell} \\ \vec{C}_{\ell} & \vec{D}_{\ell}\end{array}\right] \cdot\left[\begin{array}{l}\vec{V}_{A} \\ \vec{I}_{A}\end{array}\right]$

where the subscript " $b$ " denotes the bend discontinuities, and " $\ell$ " denotes a section of the coupled transmission lines with length $\ell$. To deembed the effects of transmission lines, a section of coupled transmission lines of straight length $2 \ell$ is considered, 
and its $A B C D$ matrix without the bend discontinuities can be formulated by

$$
\left[\begin{array}{l}
\vec{V}_{D} \\
\vec{I}_{D}
\end{array}\right]_{\text {without bend }}=\left[\begin{array}{cc}
\vec{A}_{\ell} & -\vec{B}_{\ell} \\
-\vec{C}_{\ell} & \vec{D}_{\ell}
\end{array}\right]^{-1} \cdot\left[\begin{array}{cc}
\vec{A}_{\ell} & \vec{B}_{\ell} \\
\vec{C}_{\ell} & \vec{D}_{\ell}
\end{array}\right] \cdot\left[\begin{array}{l}
\vec{V}_{A} \\
\vec{I}_{A}
\end{array}\right]
$$

The $A B C D_{\ell}$ matrix can be solved from (2) and then substituted into (1) to extract the desired $A B C D_{b}$ matrix for coupled bend discontinuities.

\section{Lumped Circuit Model Extraction}

Given the $A B C D_{b}$ matrix of coupled bend discontinuities, the corresponding S-matrix can be obtained and written as

$$
\begin{aligned}
{\left[\begin{array}{l}
b_{1} \\
b_{2} \\
b_{3} \\
b_{4}
\end{array}\right]=} & {\left[\begin{array}{llll}
\mathrm{S}_{11} & \mathrm{~S}_{12} & \mathrm{~S}_{13} & \mathrm{~S}_{14} \\
\mathrm{~S}_{21} & \mathrm{~S}_{22} & \mathrm{~S}_{23} & \mathrm{~S}_{24} \\
\mathrm{~S}_{31} & \mathrm{~S}_{32} & \mathrm{~S}_{33} & \mathrm{~S}_{34} \\
\mathrm{~S}_{41} & \mathrm{~S}_{42} & \mathrm{~S}_{43} & \mathrm{~S}_{44}
\end{array}\right]\left[\begin{array}{l}
a_{1} \\
a_{2} \\
a_{3} \\
a_{4}
\end{array}\right] } \\
= & {\left[\begin{array}{cccc}
\alpha_{11} & \alpha_{12} & 1+\alpha_{13} & \alpha_{14} \\
\alpha_{21} & \alpha_{22} & \alpha_{23} & 1+\alpha_{24} \\
1+\alpha_{31} & \alpha_{32} & \alpha_{33} & \alpha_{34} \\
\alpha_{41} & 1+\alpha_{42} & \alpha_{43} & \alpha_{44}
\end{array}\right]\left[\begin{array}{l}
a_{1} \\
a_{2} \\
a_{3} \\
a_{4}
\end{array}\right] }
\end{aligned}
$$

where $\alpha$ 's are small quantities if the size of discontinuity is much smaller than one wavelength of the operating frequency range. It is noteworthy that the $\alpha$ 's satisfy $\alpha_{i j}=\alpha_{j i}$ due to reciprocity, and in addition $\alpha_{11}=\alpha_{33}, \alpha_{22}=\alpha_{44}, \alpha_{23}=\alpha_{14}$, and $\alpha_{12}=\alpha_{34}$ due to the structural symmetry. As a result, there are only six independent $\alpha$ 's in (3).

While all the terms corresponding to higher orders of $\alpha$ 's are negligible, the $\alpha$ 's can be related to the lumped circuit elements of Fig. 1(c) in light of Kirchoff's Current Law by

$$
\begin{aligned}
\alpha_{11}-\alpha_{13} & =\frac{\left(R_{13}+j \omega L_{13, S}\right)}{Z_{0}} \\
\alpha_{22}-\alpha_{24} & =\frac{\left(R_{24}+j \omega L_{24, S}\right)}{Z_{0}} \\
\alpha_{12}-\alpha_{14} & =\frac{\left(R_{12}+j \omega L_{m}\right)}{Z_{0}} \\
-\frac{\left(\alpha_{11}+\alpha_{13}+\alpha_{12}+\alpha_{14}\right)}{2} & =\left(G_{11}+j \omega C_{11, S}\right) \times Z_{0} \\
& =\left(G_{33}+j \omega C_{33, S}\right) \times Z_{0}
\end{aligned}
$$

$$
\begin{aligned}
-\frac{\left(\alpha_{22}+\alpha_{24}+\alpha_{12}+\alpha_{14}\right)}{2} & =\left(G_{22}+j \omega C_{22, S}\right) \times Z_{0} \\
& =\left(G_{44}+j \omega C_{44, S}\right) \times Z_{0}
\end{aligned}
$$

$$
\begin{aligned}
\frac{\left(\alpha_{12}+\alpha_{14}\right)}{2} & =\left(G_{12}+j \omega C_{12, m}\right) \times Z_{0} \\
& =\left(G_{34}+j \omega C_{34, m}\right) \times Z_{0}
\end{aligned}
$$

where the subscript " $s$ " denotes the self terms, and " $m$ " denotes the mutual terms. Note that $R_{i j}$ and $G_{i j}$ are not shown

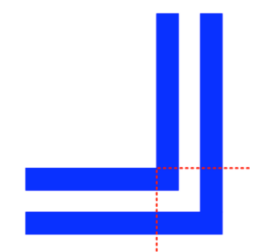

(a)

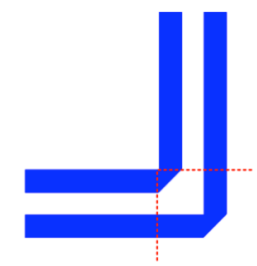

(b)

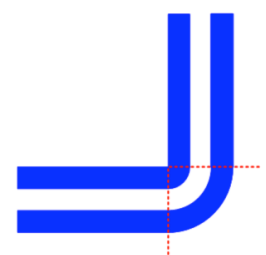

(c)

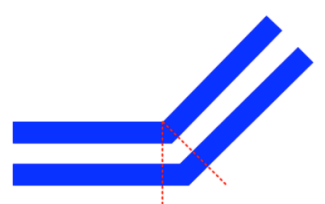

(d)

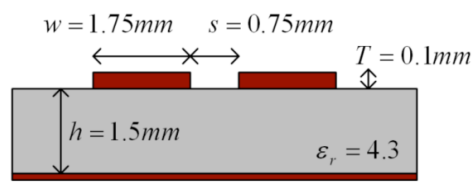

(e)

Fig. 2. Physical structures of various bent coupled transmission lines and the cross-sectional view. (a) Right-angle bends. (b) Right-angle with miter bends. (c) Round-corner bends. (d) 45-degree-angle bends. (e) Cross-sectional view of the microstrip structure.

in Fig. 1(c). Since they are usually small and can be neglected in the practical frequency range.

\section{TRANSIENT ANALYSES}

For various bent differential transmission line structures, the transient analyses using the full-wave solver IE3D at the frequency of $2 \mathrm{GHz}$ are presented in this section. These structures include the coupled right-angle bends, right-angle with miter bends, round-corner bends, and 45-degree-angle bends, while the substrate dielectric constant $\varepsilon_{r}=4.3$, height $h=1.5$ $\mathrm{mm}$, microstrip width $w=1.3 \mathrm{~mm}$, thickness $T=0.05 \mathrm{~mm}$, and center-to-center separation $s=1.8 \mathrm{~mm}$ as depicted in Fig. 2. Following the aforementioned procedures in Section II, the lumped equivalent circuit parameters corresponding to the various bend structures are extracted and summarized in Table I. 
TABLE I

EXTRACTEd PARASITIC PARAMETERs OF VARIOUS BEND STRUCTURES AS DEPICTED IN Fig. 2.

\begin{tabular}{|c|c|c|c|c|c|c|}
\hline Bend Structure & $\begin{array}{c}L_{13} \\
(\mathrm{nH})\end{array}$ & $\begin{array}{c}L_{24} \\
(\mathrm{nH})\end{array}$ & $\begin{array}{c}L_{m} \\
(\mathrm{nH})\end{array}$ & $\begin{array}{c}C_{11}=C_{33} \\
(\mathrm{pF})\end{array}$ & $\begin{array}{c}C_{22}=C_{44} \\
(\mathrm{pF})\end{array}$ & $\begin{array}{c}C_{12}=C_{34} \\
(\mathrm{pF})\end{array}$ \\
\hline Right-Angle Bent & 0.058 & 1.889 & 0.053 & 0.052 & 0.271 & 0.028 \\
\hline Right-Angle with Miter Bend & 0.139 & 2.014 & 0.011 & 0.030 & 0.248 & 0.013 \\
\hline Round-Corner Bend & 0.104 & 1.746 & 0.079 & 0.038 & 0.217 & 0.024 \\
\hline 45-Deree-Angle Bend & 0.181 & 0.961 & 0.092 & 0.024 & 0.118 & 0.017 \\
\hline
\end{tabular}

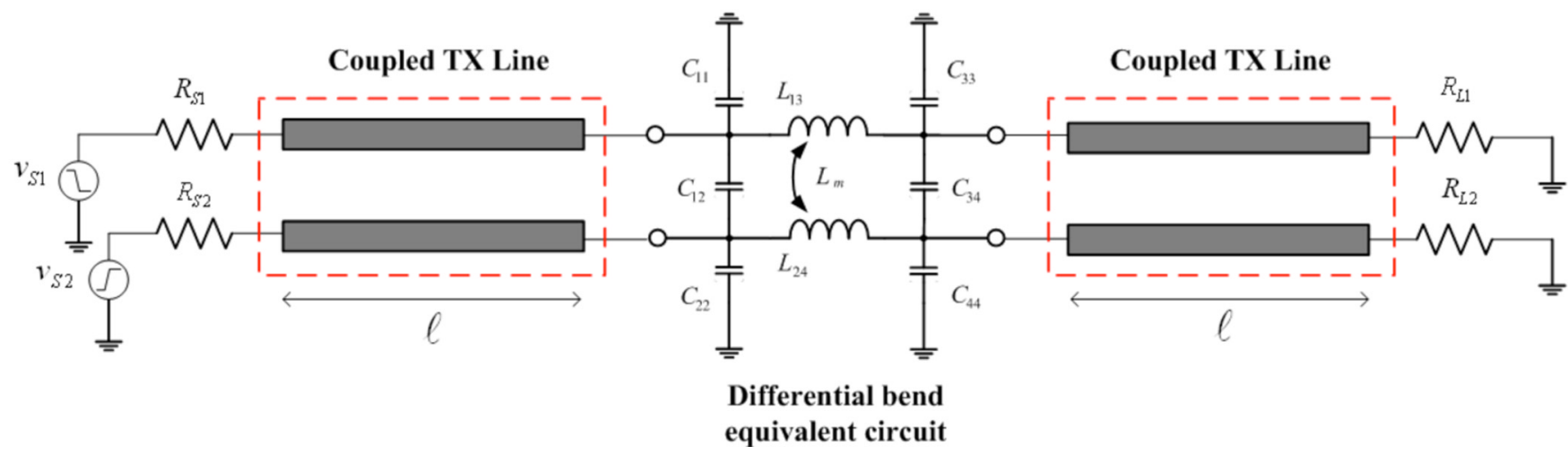

Fig. 3. Simulation structure for the bend differential transmission lines.

The $S$-parameters obtained directly from the field solver and those deduced from the extracted lumped circuit model are in good agreement, although not shown here.

Given the equivalent circuit model, it is easy to simulate the signal integrity effects due to the bend discontinuities by a circuit simulator. A simulation structure for bent differential signal lines of length $\ell=25 \mathrm{~mm}$ is depicted in Fig. 3, where the lumped equivalent circuit model is substituted for the bend discontinuities. Two drivers launch the differential ramped step signal with the magnitude of $\pm 1 \mathrm{~V}$ and rise time of $100 \mathrm{ps}$ onto the coupled transmission lines. Furthermore, each voltage source is in series with an internal odd-mode resistance of $50 \Omega$, while the other end of the signal lines are connected with matched loads.

In this paper, the common-mode voltage $V_{c}$, and differentialmode voltage $V_{d}$, are respectively defined by

$$
\left[\begin{array}{l}
V_{c} \\
V_{d}
\end{array}\right]=\left[T_{m-l}\right]\left[\begin{array}{l}
V_{1} \\
V_{2}
\end{array}\right] \equiv\left[\begin{array}{cc}
1 / 2 & 1 / 2 \\
1 & -1
\end{array}\right]\left[\begin{array}{l}
V_{1} \\
V_{2}
\end{array}\right]
$$

where $\left[T_{m-l}\right]$ is a $2, \times 2$ matrix denoting the transformation from line voltages to modal voltages. For the bent differential transmission lines, transient analyses of the common-mode noise at the receiver and the reflected differential-mode noise at the sending end are shown in Fig. 4. Because of the length mismatch, asymmetrical interconnect, and differential bend, there is a difference of time delay between the two adjacent routing traces. The differential phase skew results in the conversion from differential to common mode and then the common-mode noise is induced [13].

In reference to Fig. 4, the 45-degree-angle bend structure, therefore, has the minimum common-mode noise and reflected differential-mode noise. In contrast to the voltage fluctuation, the other three bend structures have larger noise. The reduction amount of the common-mode noise induced by the 45 -degree-angle bend is about $60 \%$ of that induced by the right-angle bend. As for the round-corner bend against the right-angle bend, its reflected differential-mode noise at the sending end can be somehow reduced, although the common-mode noise at the receiver is hardly alleviated. This is attributed to the large phase skew in comparison with that of the 45-degree-bend, though the discontinuity impedance of round-corner bend is improved.

For the signal integrity effects of various rise-time settings on the common-mode and reflected differential-mode noise, the transient analyses are shown in Fig. 5. It is found that the faster the rise-time setting of an input signal is fed, the higher both the common-mode noise and reflected differential-mode noise are observed. Empirically, while the rise time is lower than $100 \mathrm{ps}$ with its corresponding frequency at $0.35 / 100 \mathrm{ps}=3.5 \mathrm{GHz}$ for the high-speed interconnect, the design encounters significant noise at the bend discontinuities.

\section{NOISE REDUCTION SCHEME ANALYSES}

\section{A. Dual Back-to-Back Coupled Bends}

Due to the same velocity of even-mode and odd-mode signals, the stripline structure can cancel the common-mode noise but the microstrip structure cannot. A common practical routing scheme using dual back-to-back coupled bends with arbitrary angle $\theta$ from $0^{\circ}$ to $90^{\circ}$ as depicted in Fig. 6 is employed to maintain the same trace length without the significant skew.

For the same parameters of cross section as in Fig. 2(e), the simulation is performed for the differential traces with the outside length $\ell_{1}$ and inner length $\ell$ of the dual bends. A differential ramped step signal with the magnitude of $\pm 1 \mathrm{~V}$ at the rise time of $1 \mathrm{~ns}$ is used for the excitation. Consider the dual 


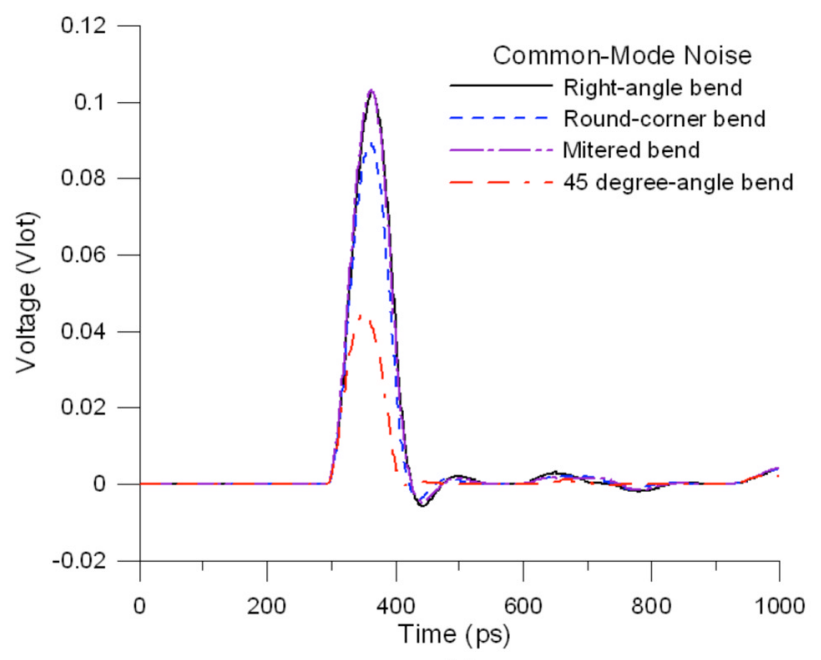

(a)

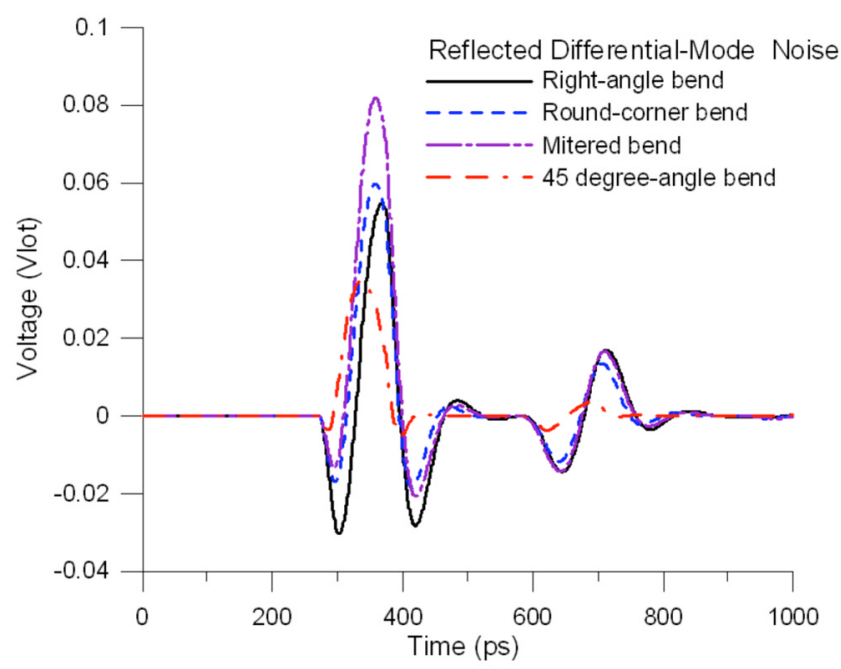

(b)

Fig. 4. Transient analyses for various bend structures at the rise time of $100 \mathrm{ps}$. (a) Common-mode noise at the receiver. (b) Reflected differential-mode noise at the sending end.

back-to-back coupled right-angle bends (when $\theta=90^{\circ}$ ) with given outside length $\ell_{1}=26 \mathrm{~mm}$ and various inner lengths $\ell=50,100,150,200,250$, and $300 \mathrm{~mm}$ as a parameter. It is found that the dual back-to-back coupled bends can help reduce the common-mode noise. The remnant common-mode noise at the probing point "g" for various length settings is shown in Fig. 7. However, the compensation becomes less effective if the length of coupled lines between the two coupled back-to-back bends increases.

While the routing length of the coupled line between dual back-to-back coupled bends increases, the velocity difference between the odd-mode and even-mode induced by the lower portion of differential bends and the remnant common-mode noise at the probing point " $\mathrm{g}$ " will increase. Nonetheless, the remnant common-mode noise will increase to become saturated until the difference in propagation time between the even-mode and odd-mode signals along the length $\ell$ is equal to twice the difference of delay time, $2 \tau=2 \times\left(T_{d 2}-T_{d 1}\right)$, between the two traces of coupled bends. Note that $T_{d 2}$ is the time delay observed on the outer corner and $T_{d 1}$ for the time delay on the inner corner

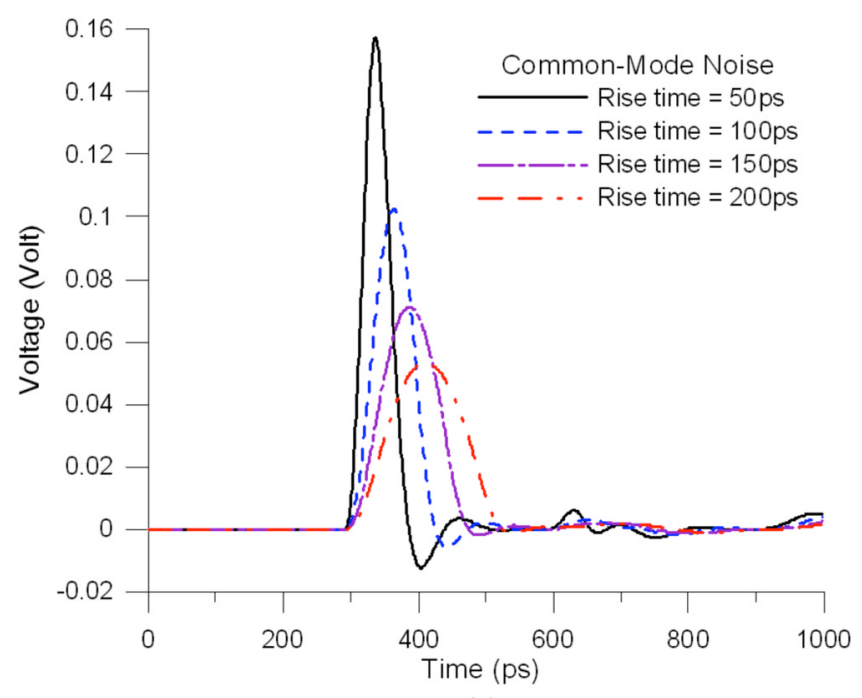

(a)

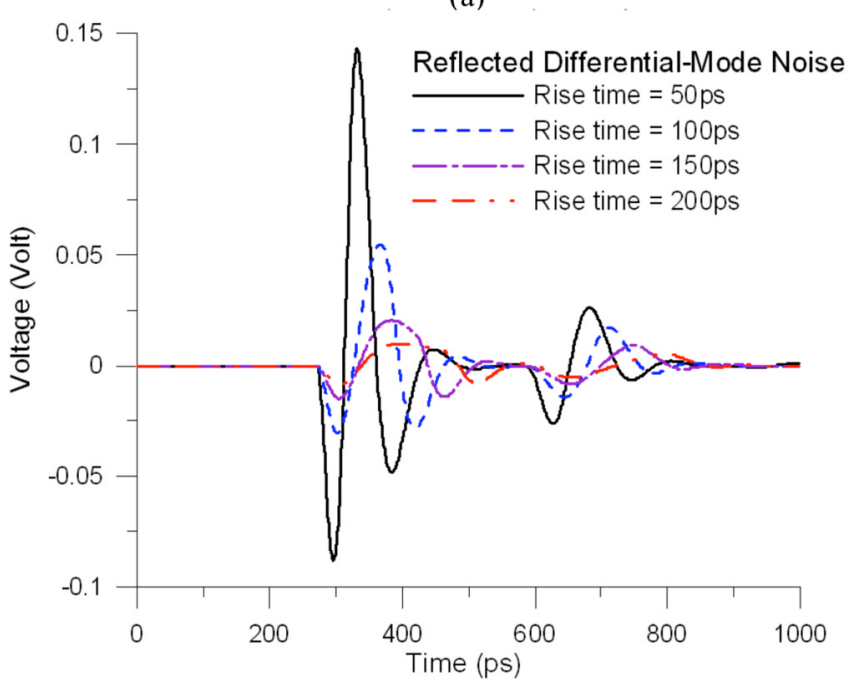

(b)

Fig. 5. Transient analyses for the right-angle bend at various rise-time settings. (a) Common-mode noise at the receiver. (b) Reflected differential-mode noise at the sending end.

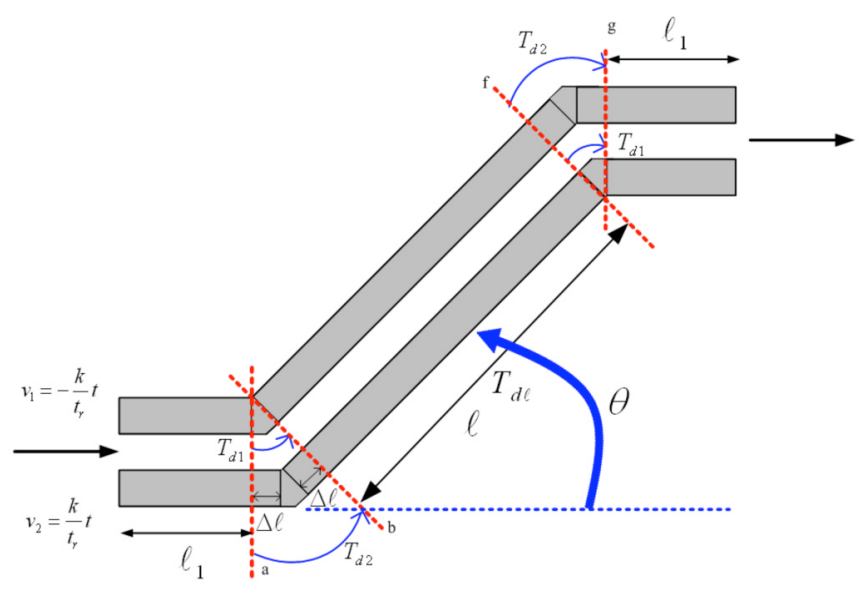

Fig. 6. Routing scheme and parameters of dual back-to-back coupled bends.

as depicted in Fig. 6. After the remnant common-mode noise has reached the saturation, a larger length $\ell$ will increase the transient width of noise. 


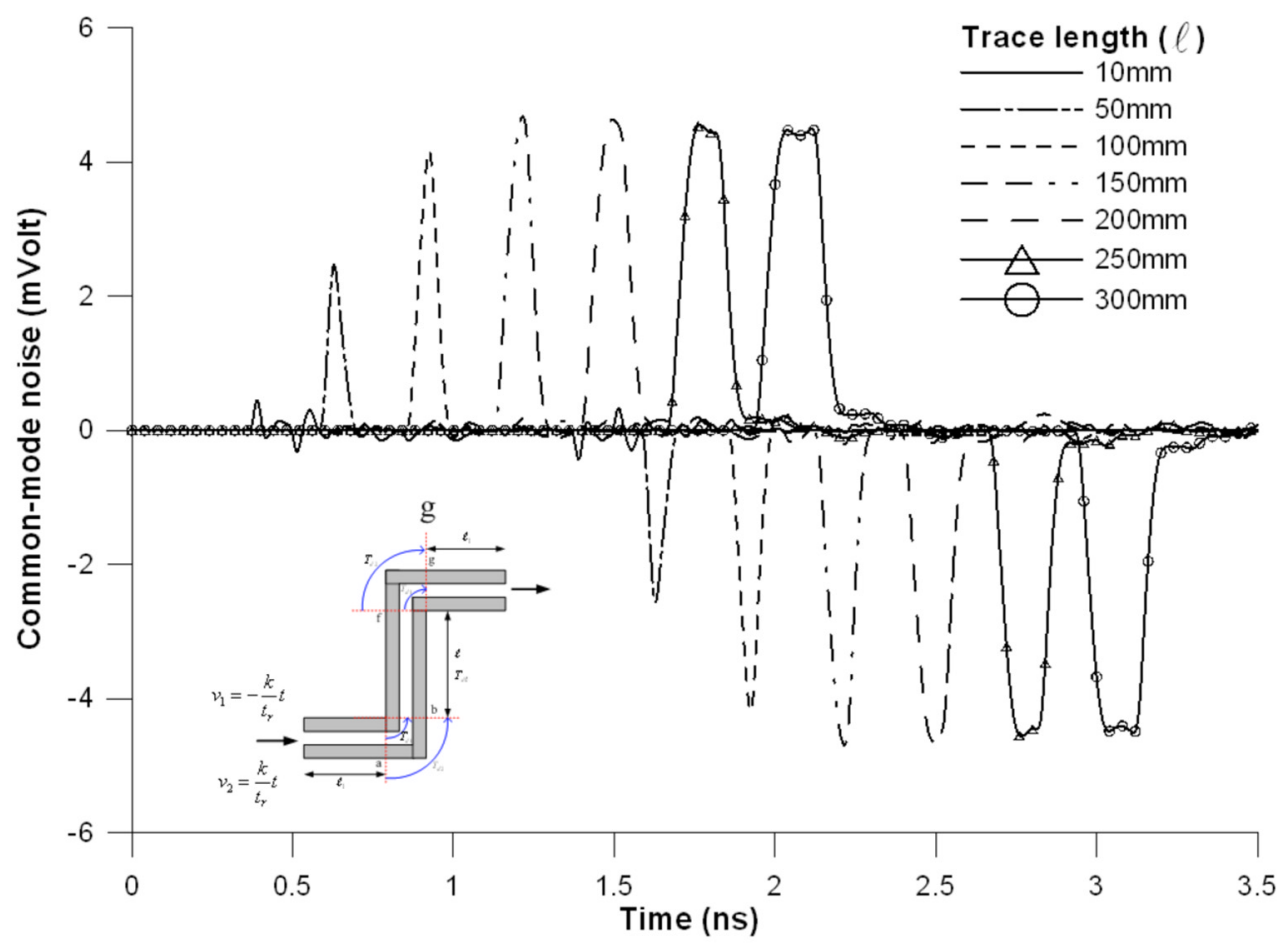

Fig. 7. Remnant common-mode noise of the dual back-to-back coupled bends observed at the probing point $\mathrm{g}$ for various length $\ell$ as depicted in Fig. 6 .

More quantitatively, let the excitation be a differential ramped step signal with magnitude $V_{\text {in }}$ and rise time $t_{r}$. The modal voltages at point "a" of Fig. 6 can be given by

$$
\begin{aligned}
{\left[\begin{array}{l}
V_{c} \\
V_{d}
\end{array}\right]_{a} } & =\left[\begin{array}{c}
0 \\
-2 V_{0}(t)
\end{array}\right] \\
V_{0}(t) & =\frac{V_{\text {in }}}{t_{r}}\left[t \cdot u(t)-\left(t-t_{r}\right) \cdot u\left(t-t_{r}\right)\right]
\end{aligned}
$$

where $u(t)$ is the unit step function. To facilitate the analysis, we neglect the effects of bend discontinuities and consider the delay effect. Cascading the effects of the bend, coupled lines, and bends following the signal trace, the modal voltage at the exit point "g" can be given by

$$
\begin{aligned}
{\left[\begin{array}{l}
V_{c} \\
V_{d}
\end{array}\right]_{g}=} & {\left[T_{m-l}\right]\left[\begin{array}{cc}
\mathcal{D}\left(T_{d 2}\right) & 0 \\
0 & \mathcal{D}\left(T_{d 1}\right)
\end{array}\right] } \\
& \times\left[T_{m-l}\right]^{-1}\left[\begin{array}{cc}
\mathcal{D}\left(T_{\text {even }}\right) & 0 \\
0 & \mathcal{D}\left(T_{\text {odd }}\right)
\end{array}\right] \\
& \cdot\left[T_{m-l}\right]\left[\begin{array}{cc}
\mathcal{D}\left(T_{d 1}\right) & 0 \\
0 & \mathcal{D}\left(T_{d 2}\right)
\end{array}\right]\left[T_{m-l}\right]^{-1}\left[\begin{array}{c}
V_{c} \\
V_{d}
\end{array}\right]_{a}
\end{aligned}
$$

where the matrix $\left[T_{m-l}\right]$ is defined in (5) and $\mathcal{D}$ is a delay operator with the delay time defined in the parenthesis. The delay times $T_{d 1}$ and $T_{d 2}$ are given in Fig. 6 , while $T_{\text {even }}=\ell / \boldsymbol{v}_{\text {even }}$ and $T_{\text {odd }}=\ell / \boldsymbol{v}_{\text {odd }}$ denote the propagation time of the common and differential modes along the inner length $\ell$, respectively.

The modal voltages at the exit point "g" can thus be related to those at the entrance point " $a$ " by the explicit matrix multiplication in (7). By applying the incident signal in (6), the coupled common mode voltage at point " $\mathrm{g}$ " can be calculated as

$$
\begin{aligned}
V_{c \text { at point } \mathrm{g}}=\frac{1}{4}\left\{\mathcal{D}\left(T_{\text {odd }}\right)-\mathcal{D}\left(T_{\text {even }}\right)\right\} \\
\cdot \cdot\left\{\mathcal{D}\left(2 T_{d 1}\right)-\mathcal{D}\left(2 T_{d 2}\right)\right\} \cdot V_{0}(t) .
\end{aligned}
$$

In common cases that $T_{b 1}, T_{b 2}<<t_{r}$, the maximum coupled common-mode noise as yielded by (8) can be given by

$$
V_{\text {comon-mode, }} \text { MAX }=\frac{V_{\text {in }}}{4 t_{r}} \cdot \min \{2 \tau, \Delta t\}
$$

where the time difference $\Delta t \equiv T_{\text {even }}-T_{\text {odd }}$ is related to the velocity difference percentage

$$
\Delta \nu(\%)=\frac{\nu_{\text {odd }}-\nu_{\text {even }}}{\nu_{\text {average }}} \cong \frac{1}{\sqrt{L_{11} C_{11}}}\left(k_{L}-k_{C}\right)
$$

by

$$
\Delta t=\frac{\ell}{\nu_{\text {average }}} \times \Delta \nu(\%)
$$

in which $v_{\text {odd }}$ and $v_{\text {even }}$ are the velocities of the common and differential modes, $v_{\text {average }}$ is their average, and $k_{C}$ and $k_{L}$ are the capacitive and inductive coupling coefficients, respectively.

It is clear from (9) that the maximum common-mode noise is proportional to the separation $\ell$ between the two bends, while it becomes saturated for larger $\ell$ such that $\Delta t>2 \tau$. In case of back-to-back coupled bends with arbitrary angle $\theta$ as depicted in Fig. 6, the difference of delay time $\tau$ in (9) is given by

$$
\tau=\frac{2 \Delta \ell}{v_{\text {average }}}=\frac{2(w+s) \cdot \tan \left(\frac{\theta}{2}\right)}{v_{\text {average }}}
$$

where $w$ and $s$ are the width and spacing of the coupled lines, respectively.

Another common practical routing scheme using a small detour for the inner trace of coupled bends, as depicted in Fig. 8, is also employed to maintain the same trace length. The analysis and results are similar to those for the dual back-to-back coupled bends. The remaining common-mode noise at point "g" will increase as the inner length $\ell$ becomes larger. However, it will become saturated when the difference in propagation time between the even-mode and odd-mode signals along the length 


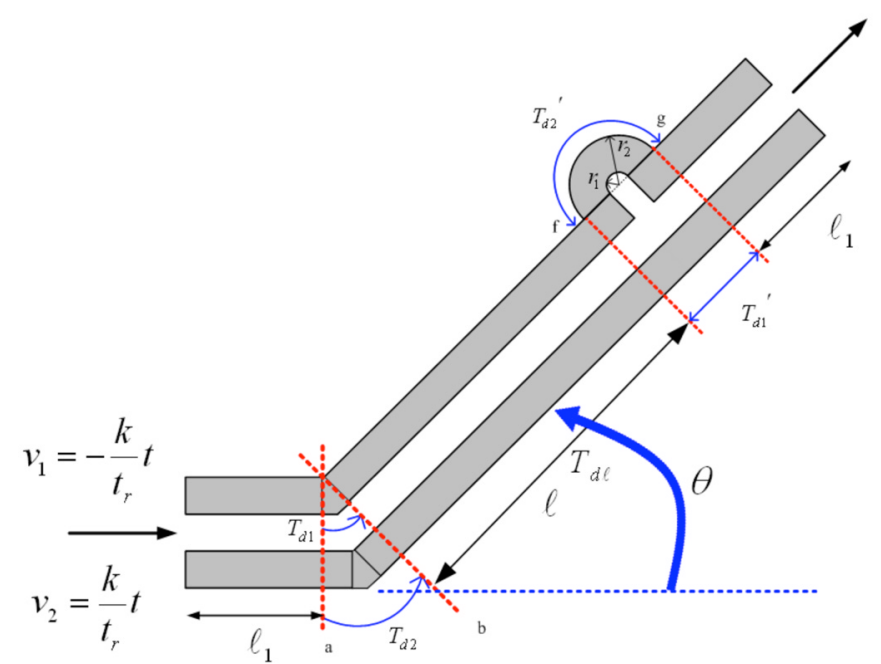

Fig. 8. Routing scheme and parameters of dual arbitrary angle bend with detour.

$\ell$ is larger than $2 \tau$. The common-mode noise at the probing " $\mathrm{g}$ " can also be calculated by formulation, as in (9).

\section{B. Compensation Capacitance for Noise Reduction}

The common-mode conversion is primarily due to the skew between the two traces of coupled bends, and, therefore, how to let the two traces have the same skew is one way to reduce the effect of common-mode conversion. An efficient way is to shunt a compensation capacitance at the bends, as shown in the inset of Fig. 9, in order to add some extra time delay for the signal along the inner short path. For the same simulation structure as in Fig. 3 with the cross-sectional parameters of Fig. 2(e), the transient common-mode noise at the receiver for various values of compensation capacitance is presented in Fig. 9.

From the scattering parameters in (3), it is not difficult to obtain the mode conversion parameter [14]

$$
S_{c 2 d 1}=\frac{1}{2}\left(S_{31}-S_{32}+S_{41}-S_{42}\right) .
$$

If suitable compensation capacitances $\left(C_{C}^{\prime}=1 / 2 C_{C}\right)$ are shunted into the two ends of the inner bend, the mode conversion parameter $S_{c 2 d 1}$ can be minimized. After some algebraic simplification, the sufficient condition for the nearly zero $S_{c 2 d 1}$ is

$$
\alpha_{13}-\alpha_{24}=0
$$

For a bend structure with compensation capacitances, formula (4) is still applicable, except that $C_{11, S}$ should be replaced by $C_{11, S}^{\prime}\left(=C_{11, S}+C_{C}^{\prime}\right)$. From (4a), (4b), (4d), and (4e), (14) can be expressed in terms of the equivalent circuit parameters and the compensation capacitance. By neglecting the small loss terms of $R_{i j}$ and $G_{i j}$, (14) can be satisfied if the compensation capacitance $\left(C_{C}\right)$ is chosen as

$$
C_{C}=2 \times\left(C_{22}+\frac{L_{24}-L_{13}}{2 Z_{\text {odd }}^{2}}-C_{11}\right)
$$

where

$$
Z_{\text {odd }}=\sqrt{\frac{L-L_{m}}{C+C_{m}}}
$$

denotes the odd-mode impedance of differential lines.

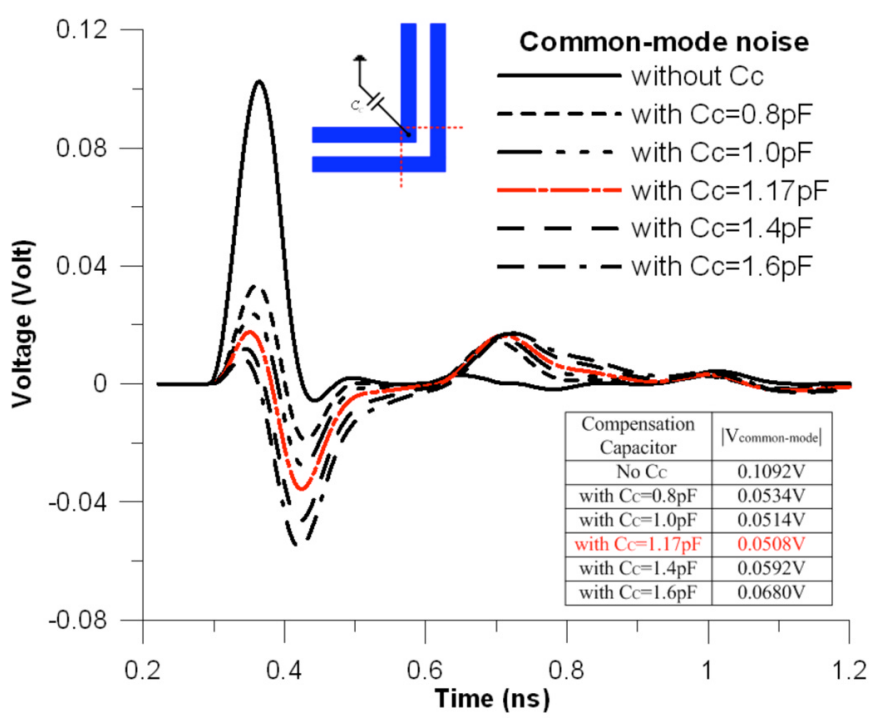

Fig. 9. Transient analyses at the receiver for right-angle bends with shunt various compensation capacitances.

For the aforementioned right-angle bend structure with $L_{13}=0.058 \mathrm{nH}, L_{24}=1.889 \mathrm{nH}, C_{11}=0.052 \mathrm{pF}$, $C_{22}=0.271 \mathrm{pF}$, and $Z_{\text {odd }}=50 \Omega$ as listed in Fig. 9, the optimal compensation capacitance can be calculated to be $1.17 \mathrm{pF}$. The simulated results in Fig. 9 validate the correctness of (15). The maximum common-mode noise versus the compensation capacitance is listed in the lower-right corner of Fig. 9 to clarify the scheme of noise reduction. It is noted that the optimal compensation capacitance of $C_{c}=1.17 \mathrm{pF}$ can reduce the maximum common-mode noise by $(0.1092 \mathrm{~V}-0.0508 \mathrm{~V}) / 0.1092 \mathrm{~V}=53.48 \%$ against that without a compensation capacitance for right-angle bend discontinuities.

Since the practical layout routing size is very small, it is difficult to shunt a discrete capacitor to the inner corner of the coupled bends. A patch metal, such as a square patch for rightangle coupled bends, as shown in the inset of Fig. 10(a), and a fan-shaped patch for 45-degree coupled bends in Fig. 10(b), act as the parallel-plate capacitance to substitute for the discrete capacitor. For the aforementioned cross-section parameters and simulation structures of coupled bends as depicted in Fig. 2, the side length of a square patch is $x=6.8 \mathrm{~mm}$ and the radius of a fan-shaped patch is $r=4 \mathrm{~mm}$, respectively, using the parallel-plate capacitance formula

$$
C_{C}=\epsilon_{0} \epsilon_{r} \frac{A}{d}
$$

for the optimal compensation capacitance of $C_{c}=1.17 \mathrm{pF}$. Here, $\varepsilon_{0}$ is the permittivity of free space, $\varepsilon_{r}$ is the substrate dielectric constant, $A$ and $d$ are the area and height of the parallel plate capacitance, respectively.

To validate the correctness of adding the parallel-plate patch and the capacitance formula (16), the equivalent circuit model parameters are extracted by employing the full-wave solver IE3D and applying the methods mentioned in Sections II and III. Then, the time- and frequency-domain simulations are performed by HSPICE and Microwave Office, respectively. The simulated waveforms for the coupled common-mode noise in Fig. 10 depict that the insertion of compensation capacitance is helpful for noise reduction. For the right-angle bends, a square 


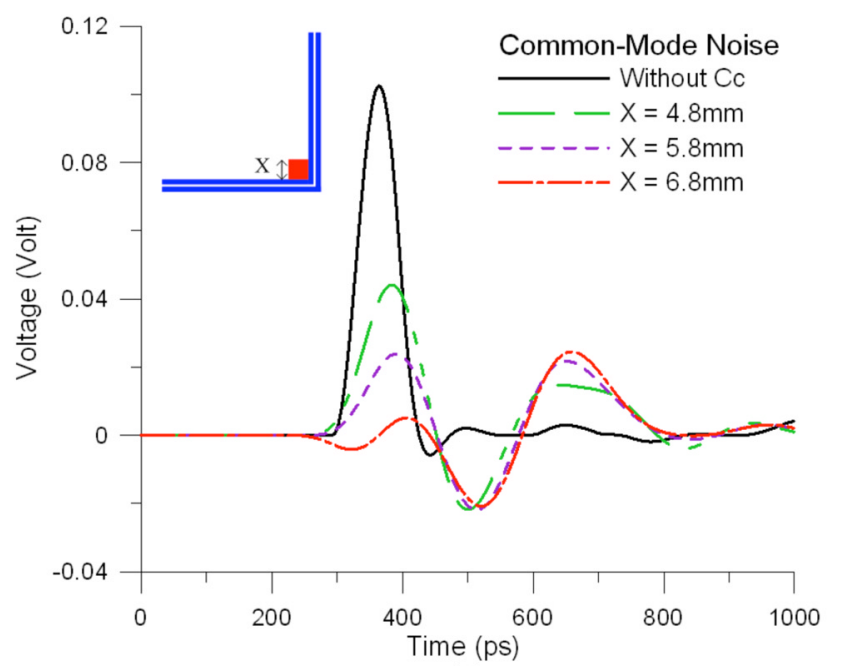

(a)

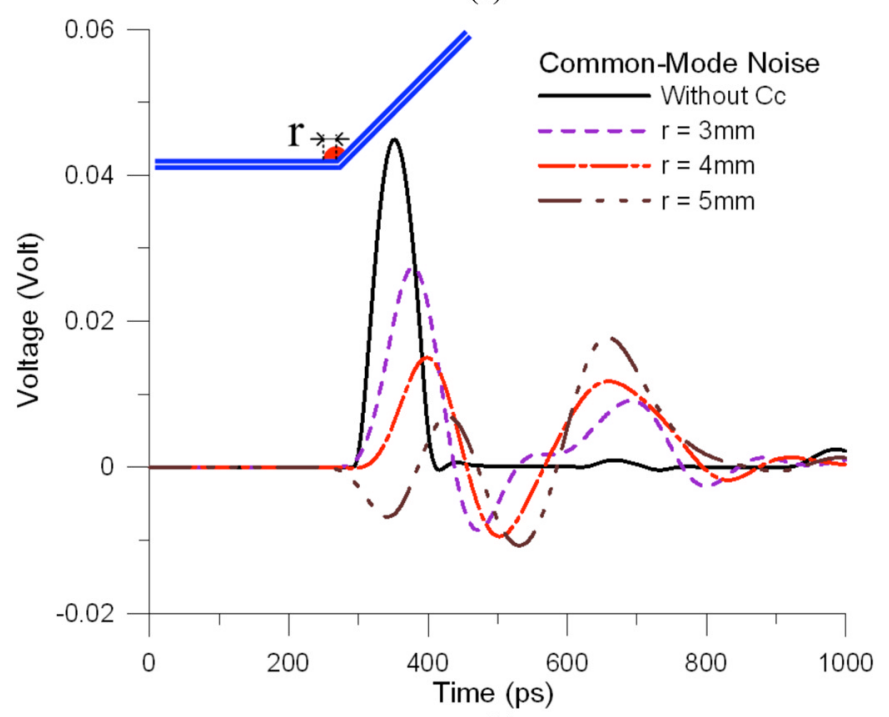

(b)

Fig. 10. Common-mode noise of coupled bends with the parallel patch capacitance. (a) Right-angle bends with a square patch. (b) 45-degree-angle bends with a fan-shaped patch.

patch of the side lengths $x=5.8 \mathrm{~mm}$ can be the acceptable choice in reference to Fig. 10(a). For 45 -degree bends with a fan-shaped patch, the radius corresponding to the maximum common-mode reduction is $r=4 \mathrm{~mm}$, as shown in Fig. 10(b). This is in good agreement with the previous prediction (16). In comparison with the noise profiles of coupled bends without a compensation capacitance, the present simple design achieves reduction in common-mode noise by $58 \%$ and $46 \%$ for rightand 45-degree angled bends, respectively.

Fig. 11(a) shows the simulated differential-to-common mode conversion parameter, $S_{c 2 d 1}$, for 45 -degree-angle bends with/without a fan-shaped patch. The reduction scheme by using a detour in Fig. 8 is also included for comparison, using parameters $\ell=4 \mathrm{~cm}, r_{1}=0.4 \mathrm{~mm}$, and $r_{2}=2.15 \mathrm{~mm}$. It can be found that both approaches, that with the detour and that with fan-shaped patch, can reduce the differential-to-common mode conversion. The reduction in mode conversion is about $10 \mathrm{~dB}$ for the detour approach. For the bend structure with a

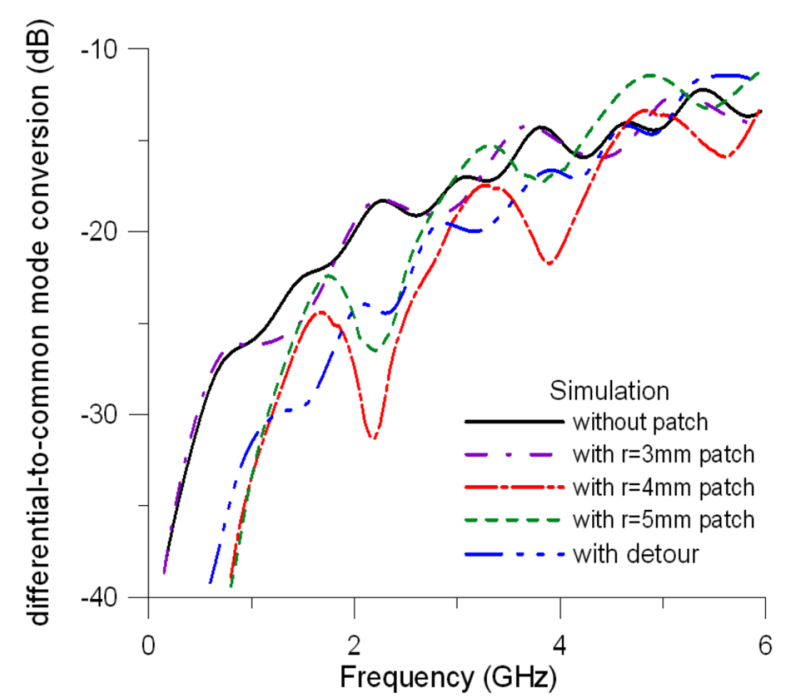

(a)

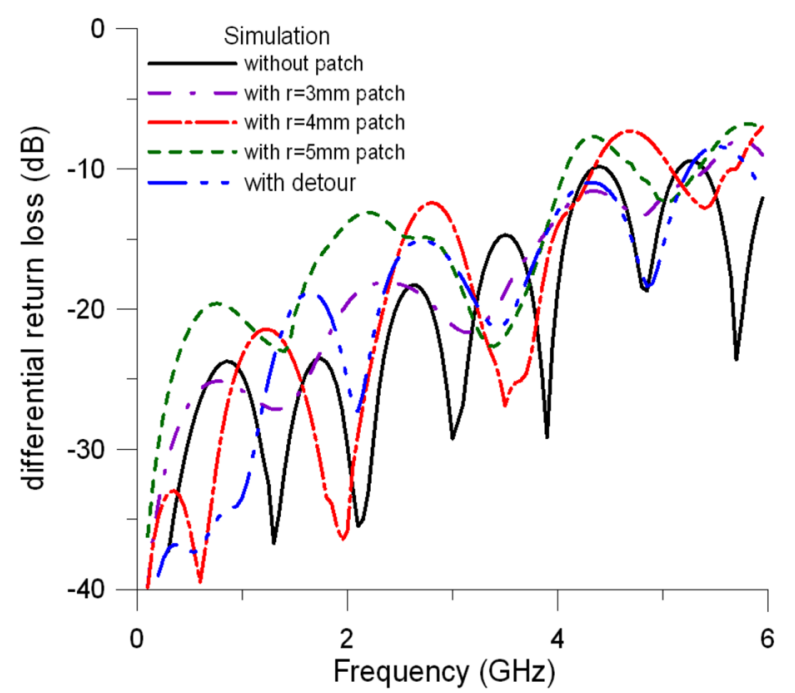

(b)

Fig. 11. Simulation results of mixed-mode $S$-parameters for 45 degree coupled bends with/without the parallel patch capacitance and with detour. (a) Differential-to-common mode conversion. (b) Differential return loss.

fan-shaped patch, the patch radius of $r=4 \mathrm{~mm}$ has the smallest mode conversion, while the noise reduction is frequency dependent.

On the other hand, neither the detour or patch compensation approach is successful in reducing the return loss for differential mode, as depicted by the simulation results in Fig. 11(b). The addition of a compensation patch may even result in a slight increase in the return loss over some frequency range. There is some tradeoff in the choice of the parallel-plate patch capacitance to reduce the differential-to-common mode conversion while not adversely affecting the differential mode return loss.

\section{EXPERIMENTAL VALIDATION}

A device under test (DUT) of coupled microstrip lines with right-angle bends as depicted in the inset of Fig. 12 was designed and fabricated to investigate the effect of differential discontinuity. The cross-sectional view and parameters of the structure 


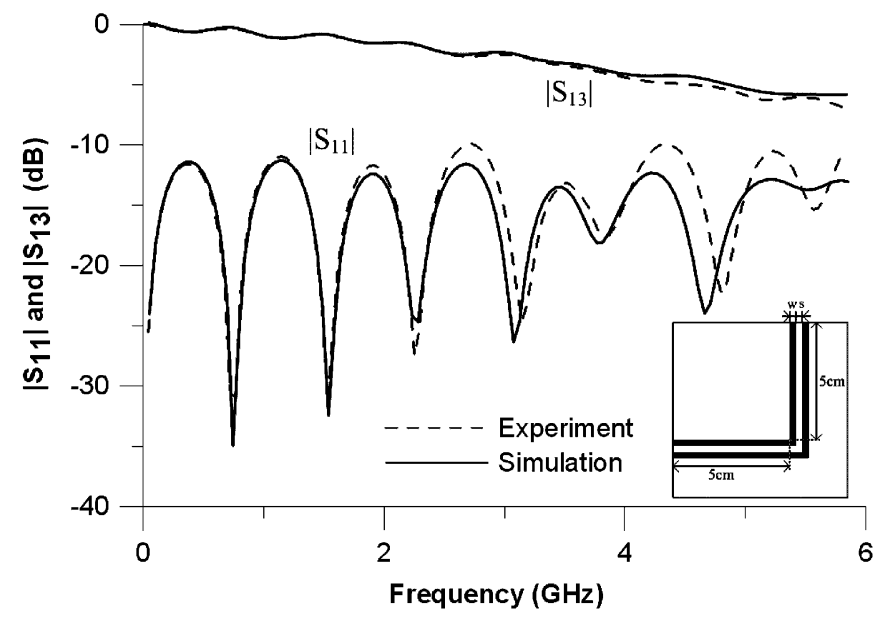

Fig. 12. Measured and simulated amplitudes of the $S_{11}$ and $S_{13}$ versus frequency for the right-angle bend discontinuities.

are stated in Fig. 2(e). As in the aforementioned methods in Sections II and III, the equivalent circuit model parameters are extracted and the simulation results in frequency-domain are obtained by using Microwave Office.

The experimental verification for DUT is performed on the frequency-domain network analyzer Agilent/E5071B. Fig. 12 shows the simulation and experimental results of $S$-parameters, which are quite closely correlated to each other in the frequency range within $4 \mathrm{GHz}$. At higher frequencies beyond $6 \mathrm{GHz}$, the equivalent circuit model for this texture will be gradually insufficient for the analysis of strongly coupled bend discontinuities. From what has been discussed above, it is clean that the consistency between the simulation and measured results validates the equivalent circuit model and analysis approach.

Following the same experimental setup, the measured results of the mixed-mode $S$-parameters for 45-degree-angle coupled bends with a fan-shaped patch of $r=4 \mathrm{~mm}$ are shown in Fig. 13. They are compared favorably with the simulation results in the frequency range within about $4 \mathrm{GHz}$, while some deviations become prominent at high frequencies due to the deficiency of the equivalent circuit model. This experiment demonstrates that insertion of a fan-shaped patch of $r=4 \mathrm{~mm}$ can significantly suppress the differential-to-common mode conversion for the 45-degree-angle coupled bends, as evident from Fig. 13(a). The resultant increase in the differential return loss is small in most frequency ranges, as shown in Fig. 13(b).

\section{CONCLUSION}

Based on the cascade property of the $A B C D$ matrix, a systematic procedure has been established to extract the lumped circuit model of bend discontinuities from the calculated full-wave $S$-parameters. The circuit model can be applied to investigate the signal integrity issues of bend discontinuities in a high-speed interconnect design by the circuit simulator. The round-corner bends can reduce the reflection at the sending end but can hardly improve the common-mode noise at the receiver. The reduction amount of the common-mode noise induced by 45 -degree-angle bends is about $60 \%$ of that induced by the right-angle bends.

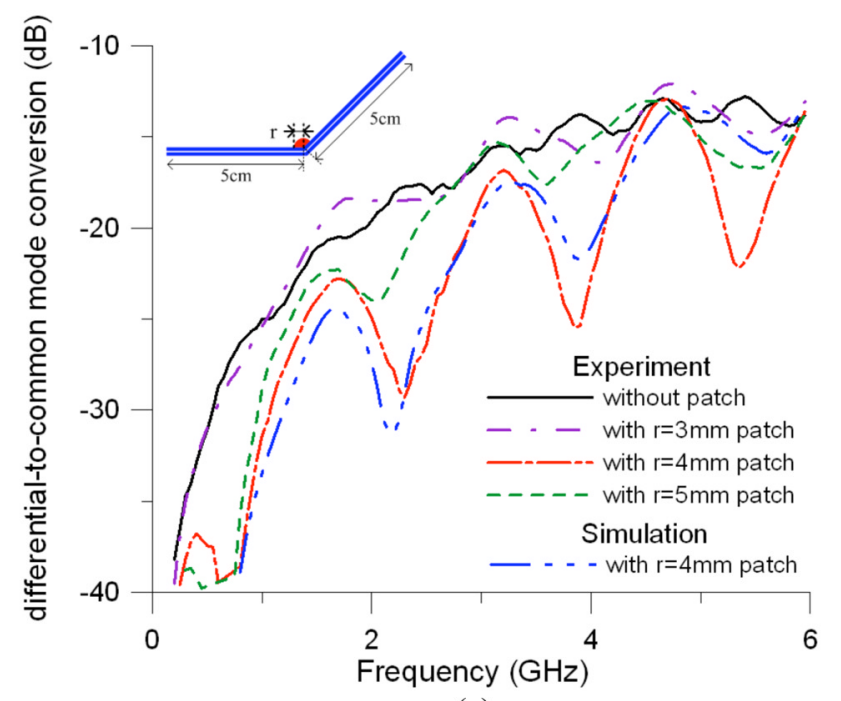

(a)

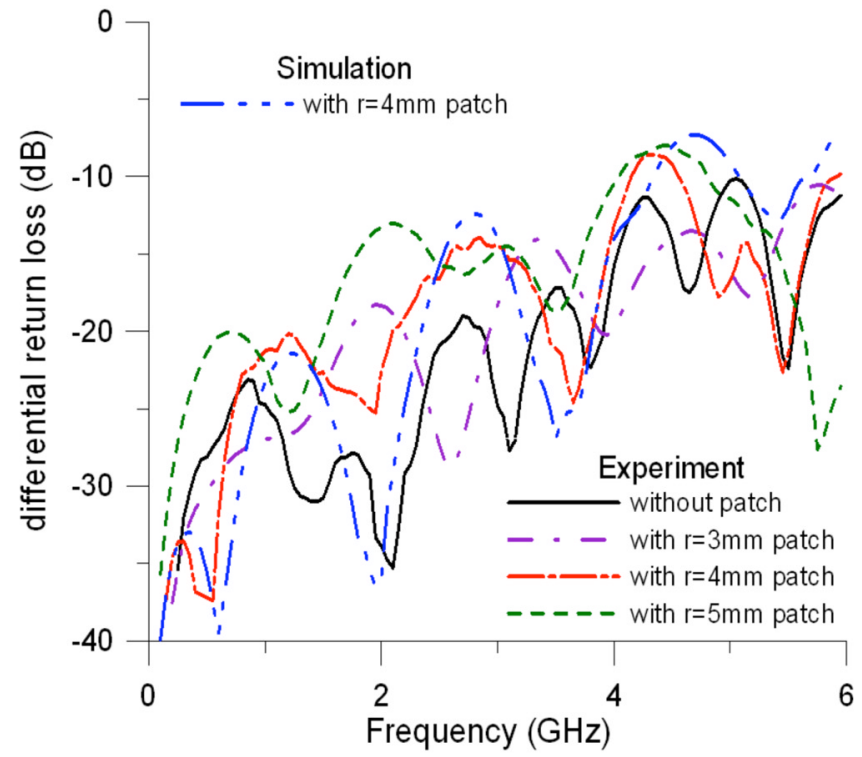

(b)

Fig. 13. Comparison of experiment and simulation results of mixed-mode $S$-parameters for 45-degree coupled bends with/without the parallel patch capacitance. (a) Differential-to-common mode conversion. (b) Differential return loss.

Note that the right-angle bends with and without miter are almost attributed to the maximum common-mode noise at the receiver. In addition, when the rise time of input signals is fed more quickly, the higher the common-mode noise is detected.

Based on the cascaded circuits, two traditional ways to reduce the common-mode noise in use of the dual back-to-back bends and bend with detour have been investigated. The compensation for noise reduction becomes less effective if the lengths of coupled lines between the dual back-to-back bends and between bend and detour increase. While the routing length increases, the remnant common-mode noise will increase and become saturated. To circumvent this routing length dependence and minimize the mode conversion parameter of bend discontinuities, a new compensation approach has been proposed and verified in this paper. The optimal value of a shunt compensation capacitance can be added so as to reduce the common-mode noise 
at the receiver. Because the routing size of a practical layout is very small, a parallel-plate patch metal is constructed to act as the compensation capacitance. Feasible schemes using a square patch for right-angle bends and a fan-shaped patch for 45-degree-angle bends are then proposed to achieve the noise reduction.

Transient analyses and mixed-mode $S$-parameters for various bend discontinuities are performed to explore the noise reduction schemes. Favorable comparisons between the simulation and measurement in frequency domain validate the equivalent circuit model, the analysis method, and the effectiveness of the compensation patch approach. The design methodology described in this paper is therefore useful for noise reduction concerned in the high-speed multigigabit per second digital circuitry.

\section{REFERENCES}

[1] R. Mehran, "Calculation of microstrip bends and Y-junctions with arbitrary angle," IEEE Trans. Microw. Theory Tech., vol. MTT-26, no. 6, pp. 400-405, Jun. 1978.

[2] N. Feix, M. Lalande, and B. Jecko, "Harmonical characterization of a microstrip bend via the finite difference time domain method," IEEE Trans. Microw. Theory Tech., vol. 40, no. 5, pp. 955-961, May 1992.

[3] G. Stewart, M. Kay, C. Riedell, R. Pomerleau, and M. Steer, "Microstrip discontinuity modeling," Proc. IEEE Energy Inf. Tech., vol. 1, pp. 107-111, Apr. 1989.

[4] A. J. Slobodnik Jr. and R. T. Webster, "Experimental validation of microstrip bend discontinuity models from 18 to $60 \mathrm{GHz}$," IEEE Trans. Microw. Theory Tech., vol. 42, no. 10, pp. 1872-1878, Oct. 1994.

[5] H. Wang, Y. Ji, T. H. Hubing, J. L. van Drewniak, T. P. Doren, and R. E. DuBroff, "Experimental and numerical study of the radiation from microstrip bends," in IEEE Int. Symp. Electromagn. Compat., vol. 2, Aug. 2000, pp. 739-741.

[6] S. Lee and M. Hayakawa, "A study on the radiation loss from a bent transmission line,” IEEE Trans. Electromagn. Compat., vol. 43, no. 4, pp. 618-621, Nov. 2001.

[7] J. L. Knighten, N. W. Smith, J. T. DiBene II, and L. O. Hoeft, "EMI common-mode current dependence on delay skew imbalance in highspeed differential transmission lines operating at 1 gigabit/second data rates," in Proc. IEEE Int. Symp. Quality Electron. Design, Mar. 2000, pp. 309-313.

[8] A. Hill and V. K. Tripathi, "Analysis and modeling of coupled right angle microstrip bend discontinuities," in IEEE MTT-S Int. Microw. Symp. Dig., vol. 3, Jun. 1989, pp. 1143-1146.

[9] F. J. Schmuckle and R. Pregla, "The method of lines for the rigorous full wave analysis of rectangular bends of multiple-line-systems," in IEEE MTT-S Int. Microw. Symp. Dig., vol. 2, Jun. 1992, pp. 797-800.

[10] P. H. Harms and R. Mittra, "Equivalent circuits for multiconductor microstrip bend discontinuities," IEEE Trans. Microw. Theory Tech., vol. 41, no. 1, pp. 62-69, Jan. 1993.

[11] J.-M. Jong, B. Janko, and V. Tripathi, "Equivalent circuit modeling of interconnects from time-domain measurements," IEEE Trans. Comp. Hybrids, Manuf. Technol., vol. 16, no. 1, pp. 119-126, Feb. 1993.

[12] C. Boussetta, F. Ndagijimana, and J. Chilo, "Modeling of multiconductor microstrip bend discontinuities," Proc. IEEE 3rd Topical Elect. Performance Electron Packag., pp. 148-150, Nov. 1994.

[13] H. Howard, H. Stephen, H. Bryce, and L. Tao, "Modeling and mitigating AC common mode conversion in multi-Gb/s differential printed circuit boards," in Proc. IEEE 13th Topical Elect. Performance Electron Packag., Oct. 2004, pp. 29-32.

[14] W. Fan, A. Lu, L. L. Wai, and B. K. Lok, "Mixed-mode $S$-parameter characterization of differential structures," in Proc. IEEE 5th Electron. Packag. Technol. Conf., Dec. 2003, pp. 533-537.

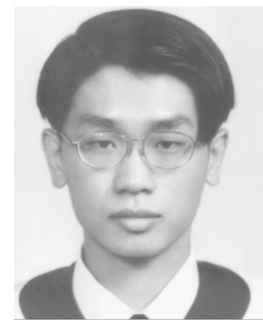

Guang-Hwa Shiue was born in Tainan, Taiwan, R.O.C., in 1969. He received the B.S. and M.S. degrees in electrical engineering from National Taiwan University of Science and Technology, Taipei, in 1995 and 1997, respectively, and the Ph.D. degree in communication engineering from National Taiwan University, Taipei, in 2006.

$\mathrm{He}$ is also currently a Lecturer in the Electronics Department, Jin Wen Institute of Technology, Taipei. His areas of interest include numerical techniques in electromagnetics, microwave planar circuits, signal/power integrity (SI/PI) and electromagnetic interference (EMI) for high-speed digital systems, and electrical characterization of system-in-package.

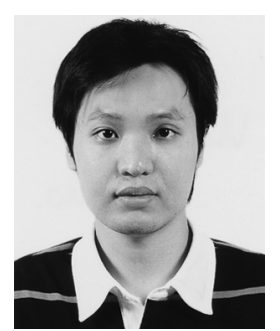

Wei-Da Guo was born in Taoyuan, Taiwan, R.O.C., on September 25, 1981. He received the B.S. degree in communication engineering from the Chiao Tung University, Hsinchu, Taiwan, in 2003, and is currently working toward the M.S. and Ph.D. degrees in communication engineering at National Taiwan University, Taipei.

His research interests include computational electromagnetics and SI/PI issues in the design of highspeed digital systems.

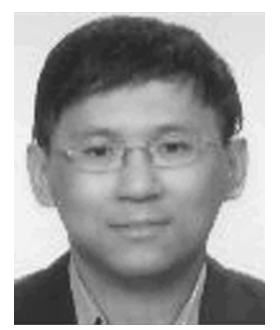

Chien-Min Lin (M'92) received the B.S. degree in physics from National Tsing Hua University, Hsinchu, Taiwan, R.O.C., the M.S. degree in electrical engineering from National Taiwan University, Taipei, Taiwan, and the Ph.D. degree in electrical engineering from The University of Washington, Seattle.

He was with IBM for the xSeries server development and Intel for the advanced platform design. Since January 2004, he has been with the Taiwan Semiconductor Manufacturing Company, Ltd., Hsinchu, as a Technical Manager in packaging design and assembly validation. He has been working on computational electromagnetics for the design applications of microwave device and rough surface scattering, signal integrity analysis for high-speed interconnect, and electrical characterization of system-in-package.

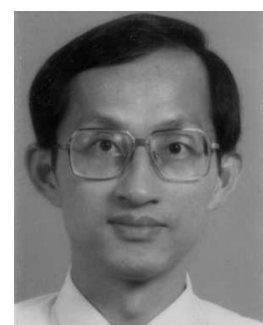

Ruey-Beei Wu (SM'97) was born in Tainan, Taiwan, R.O.C., on October 27, 1957. He received the B.S.E.E. and Ph.D. degrees from National Taiwan University (NTU), Taipei, in 1979 and 1985 , respectively.

In 1982, he joined the faculty of the Department of Electrical Engineering, NTU, where he is currently a Professor. He is also with the Graduate Institute of Communications Engineering established in 1997. From March 1986 to February 1987, he was a Visiting Scholar at the IBM East Fishkill Facility, NY From August 1994 to July 1995, he was with the Electrical Engineering Department, University of California at Los Angeles. He was appointed the Director of the National Center for High-Performance Computing from May 1998 to April 2000 and the Directorate General of the Planning and Evaluation Division from November 2002 to July 2004, both under the National Science Council. Since August 2005, he has been serving as the Chairperson of the Department of Electrical Engineering, National Taiwan University. His areas of interest include computational electromagnetics, transmission line and waveguide discontinuities, microwave and millimeter-wave planar circuits, and interconnection modeling for computer packaging. He has published more than 150 papers in international journals or conferences.

Dr. Wu is a member the Phi Tau Phi Scholastic Society and the Chinese Institute of Electrical Engineers. He served as an Associate Editor of the Journal of Chinese Institute of Electrical Engineering in 1996 and the IEEE TRANSACTIONS ON MICROWAVE THEORY AND TECHNIQUES since 2005. He was bestowed the Distinguished Research Awards by National Science Council in 1990, 1993, 1995, and 1997, and the Outstanding Electrical Engineering Professor Award by Chinese Institute of Electrical Engineers in 1999. 